\title{
Wide-bandgap InAs/InGaP quantum-dot intermediate band solar cells
}

\author{
Ínigo Ramiro, Juan Villa, Phu Lam, Sabina Hatch, Jiang Wu, Esther López, Elisa Antolín, Huiyun Liu, Antonio \\ Martí, Member, IEEE, and Antonio Luque, Senior Member, IEEE.
}

\begin{abstract}
Current prototypes of quantum-dot intermediate band solar cells suffer from voltage reduction due to the existence of carrier thermal escape. An enlarged sub-bandgap $E_{L}$ would not only minimize this problem, but would also lead to a bandgap distribution that exploits more efficiently the solar spectrum. In this work we demonstrate InAs/InGaP QD-IBSC prototypes with the following bandgap distribution: $E_{G}=1.88 \mathrm{eV}, E_{H}=1.26$ $\mathrm{eV}$ and $E_{L}>0.4 \mathrm{eV}$. We have measured, for the first time in this material, both the interband and intraband transitions by means of photocurrent experiments. The activation energy of the carrier thermal escape in our devices has also been measured. It is found that its value, compared to InAs/GaAs-based prototypes, does not follow the increase in $E_{L}$. The benefits of using thin AlGaAs barriers before and after the quantum-dot layers are analyzed.
\end{abstract}

Index Terms-quantum dots, intermediate band, solar cell characterization, intraband absorption.

\section{INTRODUCTION}

$\mathbf{T}$ HE intermediate band solar cell (IBSC) was proposed [1] as a means of harvesting solar energy conversion by allowing the use of low energy (lower than the bandgap, $E_{G}$ ) photons to produce electrical current, while maintaining a high output voltage (fundamentally limited by $E_{G}$ ). So far, most of the IBSC research has been made on quantum dot (QD) IBSCs (see Ref. [2] for a review). In such devices, the confined energy levels introduced by the QDs form the intermediate band (IB) [3] and split the matrix bandgap in two sub-bandgaps, $E_{H}$ and $E_{L}$ for the larger and smaller one, respectively.

The technologically mature InAs/GaAs QD system has been widely studied in QD-IBSCs, allowing the demonstration of the two operation principles of the IBSC: the two-photon subbandgap photocurrent [4] and the voltage preservation (voltage not limited by the IB) [5]. But it served also to evidence two main problems of this technology: the low absorption of the QDs and the existence of carrier thermal escape between the IB and the conduction band (CB). Carrier thermal escape prevents the quasi-Fermi level split between these two bands, which results in a fundamental drop of the output-voltage (that becomes limited by $E_{H}$ instead of $E_{G}$ ). This is the reason why voltage preservation could only be achieved at cryogenic temperatures, once carrier thermal escape is reduced [6], [7].

I. Ramiro, J. Villa, E. López, E. Antolín, A. Martí and A. Luque are with the Instituto de Energía Solar, Universidad Politécnica de Madrid, 28040, Madrid, Spain.

Phu Lam, Sabina Hatch, Jiang Wu and Huiyun Liu are with the Department of Electronic \& Electrical Engineering, University College London, Torrington Place, London, WC1E 7JE, UK

Manuscript received; revised.
Thermal escape is efficient at room temperature in InAs/GaAs QD-IBSC prototypes because the energy difference between the IB and the $\mathrm{CB}, E_{L}$, is small, typically lower than $0.2 \mathrm{eV}$ [8]-[10]. In light of this, QD-IBSCs where the matrix material is a wide-bandgap semiconductor were suggested [11], [12] in order to achieve a larger $E_{L}$, necessary for practical IBSCs working at room temperature. QDIBSCs using the InAs/ $\mathrm{Al}_{0.25} \mathrm{Ga}_{0.75}$ As system were fabricated showing a remarkable increase of the activation energy of the carrier thermal escape [12]. Furthermore, devices where voltage preservation has been demonstrated at temperatures as high as $260 \mathrm{~K}$ have been recently achieved [13].

Enlarging the host material bandgap and the two subbandgaps has a second beneficial effect. The optimum values for $E_{G}, E_{H}$ and $E_{L}$ are $1.95,1.24$ and $0.71 \mathrm{eV}$, respectively. Therefore, changing from GaAs, where $E_{G}=1.42$ $\mathrm{eV}$, to wider bandgaps leads to higher limiting conversion efficiencies. In Ref. [13], the values, $E_{G}=1.7 \mathrm{eV}, E_{H}=$ $1 \mathrm{eV}$ and $E_{L}=0.5 \mathrm{eV}$ are experimentally obtained in InAs/ $\mathrm{Al}_{0.25} \mathrm{Ga}_{0.75} \mathrm{As} \mathrm{QD}-\mathrm{IBSC}$ prototypes. The difference between $E_{G}$ and $\left(E_{H}+E_{L}\right)$ is identified as the value of the valence band offset (VBO), formed by the highly packed hole states in the VB of the QDs.

Theoretical calculations pointed to the $\mathrm{InAs} / \mathrm{In}_{0.3} \mathrm{Ga}_{0.7} \mathrm{P}$ QD system, grown on GaAs, as a candidate material for implementing wide-bandgap IBSCs with an improved bandgap distribution compared to the InAs/GaAs case [14]. There are some studies on InAs QDs grown on $\operatorname{In}_{0.49} \mathrm{Ga}_{0.51} \mathrm{P}$ latticedmatched to GaAs, grown by solid-source molecular beam epitaxy [15], [16] or by vapor phase epitaxy [17]. It has been found that there is intermixing between the $\mathrm{InGaP}$ spacers and the InAs QDs, leading to the presence of InGaAsP alloys, which modify the optical properties of the dots [15]. In Ref. [17] the QDs resulted larger than the typical size for InAs/GaAs, which is an undesirable effect for IBSC purposes [18]. To avoid intermixing and increase the electron confinement in the dots, in Refs. [15] and [16] thin GaAs barriers were grown before and after the QD-layers. In [19] InGaP-based $\mathrm{In}_{0.4} \mathrm{Ga}_{0.6} \mathrm{As} / \mathrm{GaAs} \mathrm{QD}$-IBSC prototypes were reported and it was discussed how these devices outperform their GaAs-based counterpart in some aspects.

In this work we have fabricated and characterized widebandgap InAs/InGaP QD-IBSC prototypes with thin AlGaAs barriers. We have measured, for the first time in this material, both the interband $(\mathrm{VB} \rightarrow \mathrm{IB})$ and the intraband $(\mathrm{IB} \rightarrow \mathrm{CB})$ transitions, obtaining a close-to-optimum bandgap distribution. We have also measured the activation energy of the thermal 
escape of carriers in our devices.

\section{EXPERIMENTAL}

\section{A. Samples fabrication}

Two samples were fabricated by solid source molecular beam epitaxy. Fig. 1 details the semiconductor $n-i-p$ structure of sample S1. The i-layer contains a stack of 20 InAs QD-layers separated by 50 nm-thick $\operatorname{In}_{0.49} \mathrm{Ga}_{0.51} \mathrm{P}$ spacers. In addition, a $3 \mathrm{~nm} \mathrm{Al}_{0.33} \mathrm{Ga}_{0.67} \mathrm{As}$ barrier and a $6 \mathrm{~nm}$ $\mathrm{Al}_{0.33} \mathrm{Ga}_{0.67} \mathrm{As}$ barrier were grown below and above the QDs. The InAs deposition for the QD formation was 2.1 MLs. All the layers were grown at $495 \mathrm{C}$ measured from a pyrometer. After capping each layer of InAs QDs with 6 $\mathrm{nm} \mathrm{Al}_{0.33} \mathrm{Ga}_{0.67} \mathrm{As}$, the substrate temperature was increased to $600{ }^{\circ} \mathrm{C}$ and annealed for $2 \mathrm{~min}$ to suppress threading dislocation formation [20]. The first and last QD-layers are separated by $100 \mathrm{~nm}$-thick $\operatorname{In}_{0.49} \mathrm{Ga}_{0.51} \mathrm{P}$ layers from the $\mathrm{p}$ - and n-emitters, respectively, in order to prevent carrier tunneling from the QDs to the CB in the emitters [7], [21]. The p-emitter consists of a $500 \mathrm{~nm}$-thick $\operatorname{In}_{0.49} \mathrm{Ga}_{0.51} \mathrm{P}$ layer doped with $\mathrm{Be}\left(1 \times 10^{17} \mathrm{~cm}^{-3}\right)$. The n-emitter consists of a 200 nm-thick $\operatorname{In}_{0.49} \mathrm{Ga}_{0.51} \mathrm{P}$ layer doped with $\mathrm{Si}\left(2 \times 10^{18} \mathrm{~cm}^{-3}\right)$. On top and beneath the $\mathrm{n}-$ and $\mathrm{p}$-emitters, respectively, 30$\mathrm{nm}$ thick $\mathrm{In}_{0.50} \mathrm{Ga}_{0.33} \mathrm{Al}_{0.17} \mathrm{P}$ layers were grown. These layers are intended to act as window (Si-doped, $5 \times 10^{18} \mathrm{~cm}^{-3}$ ) and back surface field (BSF) (Be-doped, $5 \times 10^{17} \mathrm{~cm}^{-3}$ ) layers, respectively, minimizing the surface recombination velocity. To allow the formation of the front ohmic contact, a $50 \mathrm{~nm}$ thick GaAs contact layer ( $\mathrm{Si}-$ doped, $5 \times 10^{18} \mathrm{~cm}^{-3}$ ) was grown on top of the window layer. The whole structure was grown on a (100) p-GaAs substrate. Sample S2 shares the structure of $\mathrm{S} 1$, except for the $\mathrm{i}$-layer. Instead of the QD-stack, an equally thick $\mathrm{In}_{0.49} \mathrm{Ga}_{0.51} \mathrm{P}$ layer was grown. $\mathrm{S} 2$ will perform as the reference cell in this work.

Standard photolitography techniques were used for processing the devices. The front $\mathrm{n}$-contact deposition consisted of subsequent $\mathrm{AuGe} / \mathrm{Ni} / \mathrm{Au}$ layers. After deposition of the metals, samples were submitted to a rapid thermal annealing process at $370{ }^{\circ} \mathrm{C}$. The back p-contact was then made by depositing $\mathrm{Cr}-\mathrm{Au} / \mathrm{Au}$ layers. The $50 \mathrm{~nm}$ highly-doped GaAs contact layer was not removed after the metalization process.

\section{B. Results}

Fig. 2 shows the semi-log plot of the external quantum efficiency (EQE) of samples S1 (solid line) and S2 (dotted line). The values of the InGaP bandgap, the GaAs bandgap and $E_{H}$ are indicated by vertical dashed lines. The suprabandgap EQE of S2 is below 0.4 for all photon energies. This is is due to a non-optimized structure (layers thickness, absence of anti-reflection coating layer, and presence of the absorbing contact layer). For below bandgap energies, the EQE falls drastically, but is still measurable until the GaAs bandgap energy. This sub-bandgap EQE reveals the existence of photocurrent originating from the GaAs substrate, which indicates that the BSF layer does not completely disconnect the solar cell structure from the low bandgap substrate [22]. Moreover, the oscillations in the sub-bandgap QE suggest an

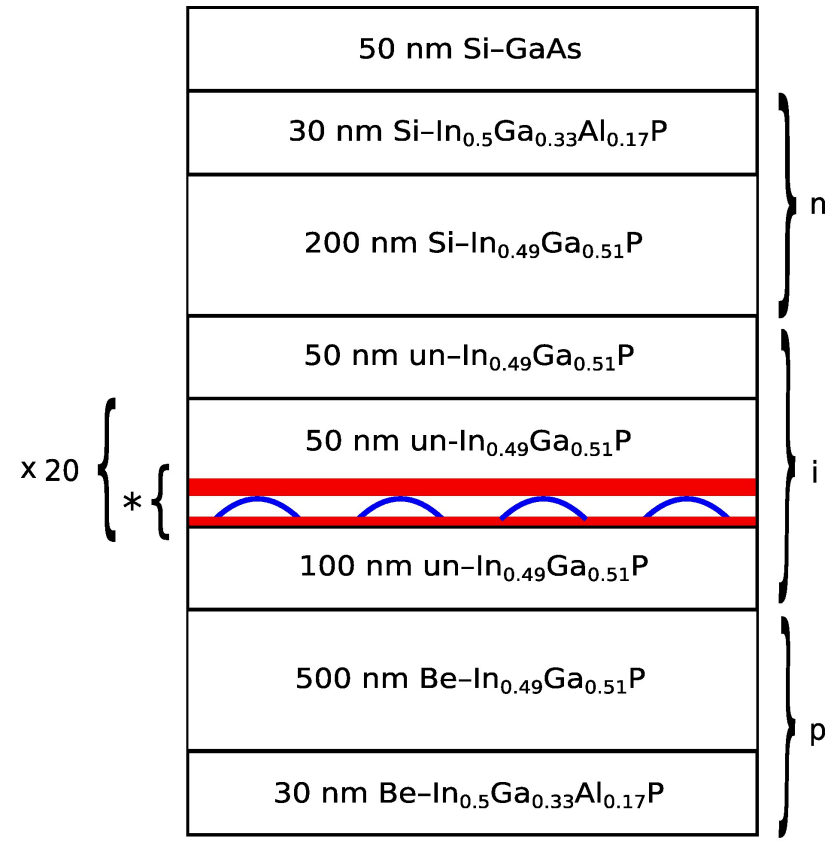

*InAs QDs with thin un- $\mathrm{Al}_{0.33} \mathrm{Ga}_{0.67} \mathrm{As}$ barriers (present only in sample S1).

Figure 1: Semiconductor structure of samples S1 (QD-IBSC prototype) and S2 (reference cell).

interference effect that is probably due to the combination of thin layers of different materials within the solar cell.

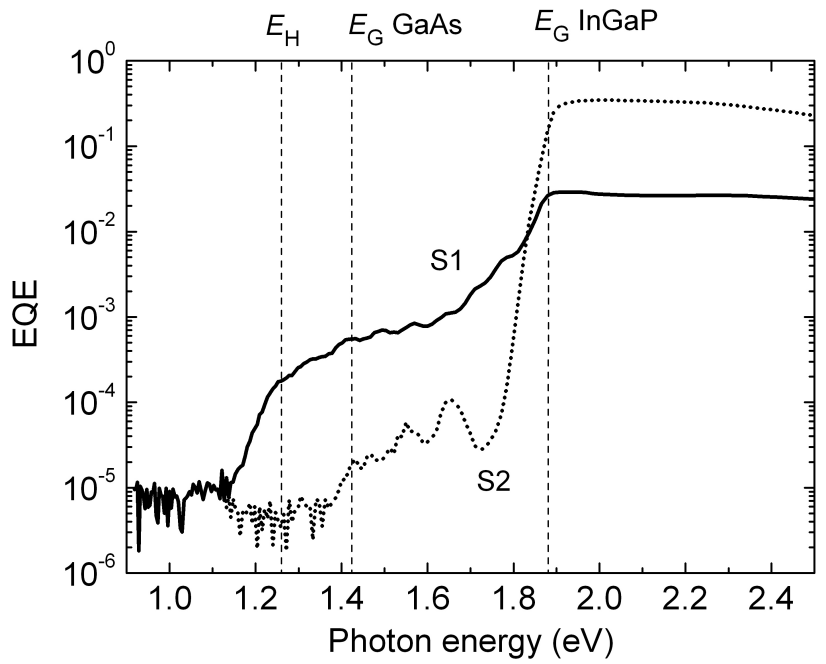

Figure 2: EQE of samples S1 (solid line) and S2 (dotted line) measured at room temperature. The values of the InGaP bandgap, the GaAs bandgap and $E_{H}$ are indicated by vertical dashed lines.

Looking now at $\mathrm{S} 1$, it can be seen that the sub-bandgap EQE extends down to approximately $1.26 \mathrm{eV}$. This value is identified as the transition between confined ground states (GSs) for holes and electrons in the QDs, $E_{H}$. This value has been confirmed by photoluminescence (PL) measurements at room temperature (shown in Fig. 5). The sub-bandgap EQE of this sample is affected by an optical interference effect as well. 
This makes it harder to identify the energies of the electronic transitions from the VB of the QDs to the confined excited states for electrons. The energy corresponding to the bandgap of the InAs wetting layer - a thin bi-dimensional layer that accompanies QDs grown in the Stransky-Krastanov mode cannot be clearly resolved either.

The supra-bandgap EQE of sample $\mathrm{S} 1$ is reduced by an order of magnitude compared to that of the reference cell. This reduction can be originated by a degradation of the electronic properties of the material, specially of the emitter [23], due to strain dislocations or defects resulting from the presence of the QDs.

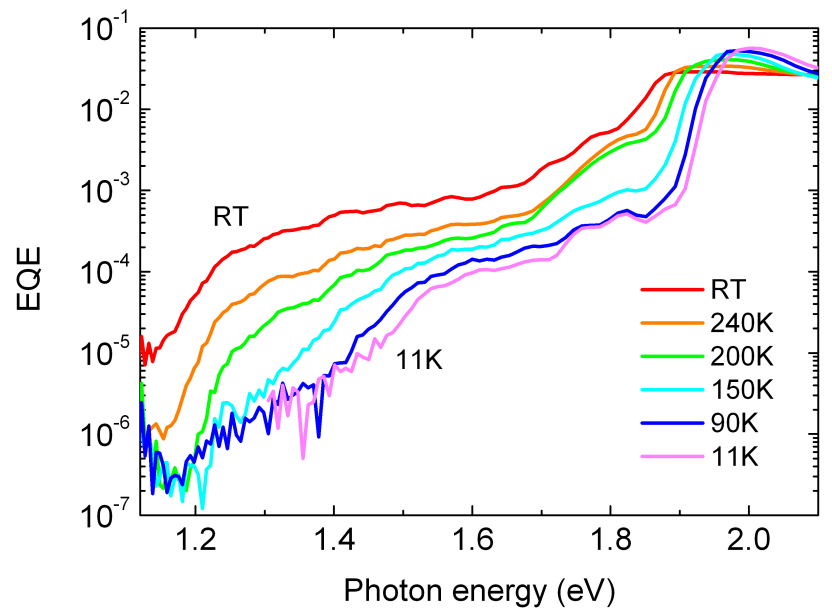

Figure 3: EQE of sample $\mathrm{S} 1$ measured at different temperatures. The sub-bandgap EQE decreases monotonically from RT to $11 \mathrm{~K}$.

Sample S1 was introduced in a closed-cycle He cryostat. Fig. 3 shows the EQE of sample S1 for different temperatures: from room temperature (RT) down to $11 \mathrm{~K}$. Focusing on the sub-bandgap part on the graph, it is seen that the EQE diminishes rapidly with decreasing temperature. This is because carrier thermal escape is inhibited as the temperature lowers, and tunnel escape is weak thanks to the thick spacers [7]. From the temperature dependency of the QE at the energy of the GSs transition, the activation energy of the carrier thermal escape can be measured [24]. This thermal process allows the detection of the sub-bandgap photocurrent in the $\mathrm{QE}$ measurement. The QE has an Arrhenius-like dependency with temperature: $\mathrm{QE} \propto \exp \left[-E_{a} /(k T)\right]$, where $E_{a}$ is the activation energy, and $k$ is the Boltzmann constant. From the slope of the $\ln (\mathrm{QE}), E_{a}$ can be obtained. The result is presented in Fig. 4 (top). The slope, corresponding to $E_{a}=$ $118 \mathrm{meV}$, was the best fitting to the measurements.

Fig. 5 shows the temperature dependence of the PL spectrum of sample S2. A 532nm continuous wave-diode-pumped solid-state laser was used to excite the sample and the signal was picked up by a TE-cooled germanium photodetector in conjunction with a monochromator with lock-in amplifier. The sample was mounted inside a close-cycled cryostat subjected to circulating liquid helium. The excitation power density was approximately $10 \mathrm{~W} \mathrm{~cm}^{-1}$. In the RT measurement (red line) two peaks at $1.24 \mathrm{eV}$ and $1.29 \mathrm{eV}$, approximately, can be
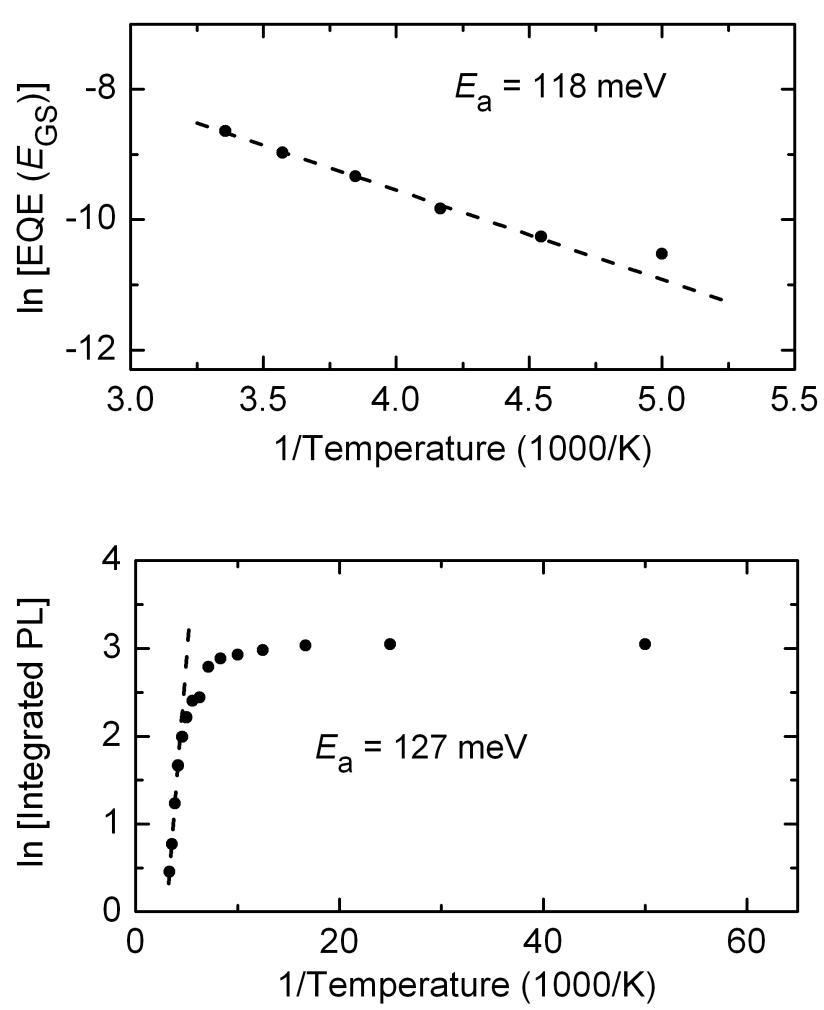

Figure 4: Measured activation energy of the thermal carrier escape in sample S1 obtained by (top) EQE measurements and (bottom) PL measurements.

resolved. As the temperature is lowered, the measured signal increases monotonically and new higher-energy peaks, related to recombination from confined excited states, appear. For temperatures below $80 \mathrm{~K}$, a peak is resolved at around $1.57 \mathrm{eV}$. The origin of this peak is not clear. It is probably due to high energy excited states in the QDs, although we cannot discard at this stage that it comes from recombination in the InAs wetting layer. At very low temperatures, the $\mathrm{InGaP}$ emission $(1.94 \mathrm{eV})$, originated at the front emitter or at the spacers, is detected.

The activation energy of the carrier thermal escape can be obtained also by fitting the temperature dependency of the PL to an Arrhenius equation. Contrarily to the $\mathrm{QE}$ case, the thermal excitation of carriers out of the potential well of the QDs does not favor, but instead reduces, the PL originated at the QDs. Carrier escape diminishes the radiative recombination between confined states (IB $\rightarrow \mathrm{VB}$ ) in favor of recombination (radiative or non-radiative) in the host material $(\mathrm{CB} \rightarrow \mathrm{VB})$. The integrated $\mathrm{IB} \rightarrow \mathrm{VB}$ QD PL intensity is proportional to $\exp \left[E_{a} /(k T)\right]$. The best fitting is obtained for $E_{a}=127$ $\mathrm{meV}$, and is shown in Fig. 4 (bottom). Both values, obtained using the EQE and PL measurements, are close, but the fitting quality was better for the PL case.

Using a $\mathrm{SiC}$ infrared lamp and a triple-grating monochromator, we were able to measure the photocurrent produced in sample S1 by mid-infrared illumination, as shown in Fig. 6. The detection was made by standard lock-in techniques. A proper set of optical filters was used to prevent undesired light existing the monochromator from reaching the sample. 


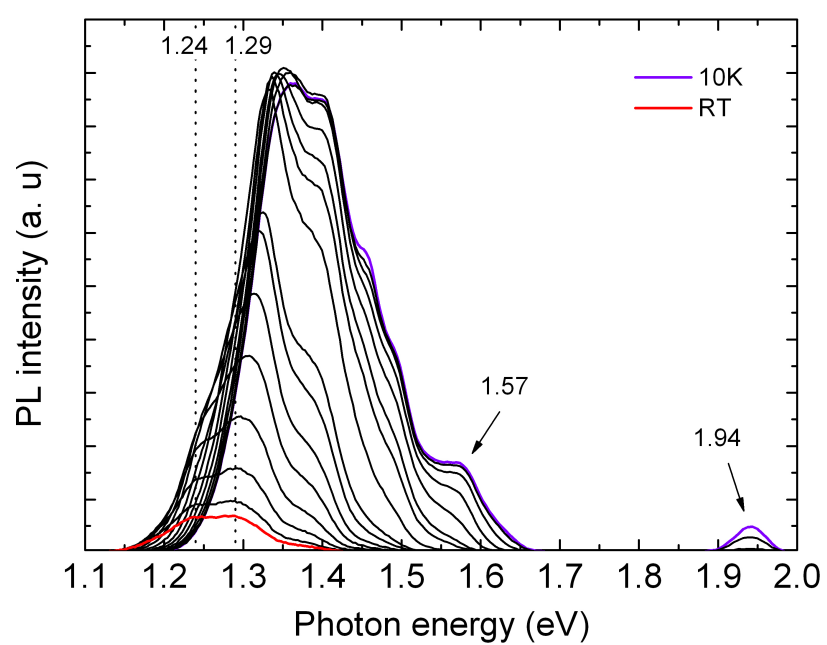

Figure 5: PL at different temperatures of sample S1. The excitation power was approximately $10 \mathrm{~W} \mathrm{~cm}^{-1}$. The measurements at RT and $10 \mathrm{~K}$ are highlighted with red and violet colors, respectively.

The sample was biased at $0 \mathrm{~V}$ and the frequency of the lock-in detection was $337 \mathrm{~Hz}$. No signal could be detected at RT. However, at low temperatures there is a clear photoresponse peak in the range $0.4-0.6 \mathrm{eV}$, as shown in the figure for the measurement at $70 \mathrm{~K}$. This photocurrent is identified as originating from the QDs, corresponding to absorption promoting $\mathrm{IB} \rightarrow \mathrm{CB}$ transitions. To our knowledge, this is the first time that the intraband transition is measured in InAs/InGaP QDs.

It is surprising that photocurrent was produced under illumination with photons capable only of promoting electrons from the IB to the $\mathrm{CB}$. In this respect, electrons pumped from the IB to the $\mathrm{CB}$ exit the cell at the $\mathrm{n}$-contact and re-enter the cell at the VB of the p-contact. Therefore, in order to return to the IB and close the circuit, there should exist some leakage mechanism that allows the electrons to return to the IB from the VB. In Refs. [13], [25] a second light beam, whose photons had enough energy to optically produce this $\mathrm{VB} \rightarrow \mathrm{IB}$ transition, was used to allow the infrared-related photocurrent detection.

\section{DISCUSSION}

The EQE and infrared photocurrent results allow us to determine the bandgap distribution of our device. Fig. 7 (left) will be used as support for the discussion. From the $\mathrm{EQE}$ at RT, the values $E_{G}=1.88 \mathrm{eV}$ and $E_{H}=1.26$ $\mathrm{eV}$ are obtained. According to Anderson's model [26], the CB discontinuity, $\Delta E_{C}$, between the $\mathrm{Al}_{0.33} \mathrm{Ga}_{0.66}$ As and the $\mathrm{In}_{0.49} \mathrm{Ga}_{0.51} \mathrm{P}$ should be around $0.4 \mathrm{eV}$ (the difference in electron affinities), however, a value of $108 \mathrm{meV}$ has been experimentally obtained for $\mathrm{Al}_{0.33} \mathrm{Ga}_{0.66} A s / \mathrm{In}_{0.49} \mathrm{Ga}_{0.51} \mathrm{P}$ interfaces [27]. We will use this value, due to the lack of more accurate ones, for sketching the band diagram of our device even if in our case the AlGaAs layer is not bulk, but only a few nanometers thick. The VB discontinuity is, then, easily calculated as $\Delta E_{V}=E_{G}+\Delta E_{C}-E_{B}=148$

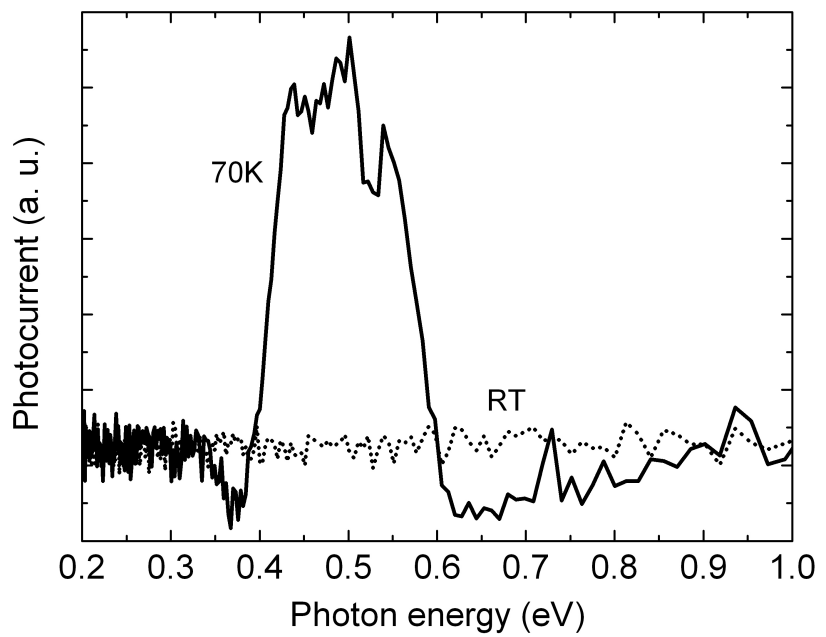

Figure 6: Photocurrent response to mid-infrared illumination of sample S1 at RT (dotted line) and $70 \mathrm{~K}$ (solid line). The measurement was performed under short-circuit conditions. The lock-in detection frequency was $377 \mathrm{~Hz}$.

meV, where $E_{B}$ is the bandgap of the AlGaAs barriers. The infrared photocurrent has its onset at around $0.4 \mathrm{eV}$ and decays abruptly at around $0.6 \mathrm{eV}$. Unfortunately, due to the small signal-to-noise ratio of the measurement, we cannot identify whether it is produced by absorption between two states only or more than two are involved. To stay in the safe-side and not overestimate $E_{L}$, it is fair to assume that the minimum value for $E_{L}$ is $0.4 \mathrm{eV}$. Although this value has been measured at $70 \mathrm{~K}$, we assume that it remains the same at RT. In fact, in our measurements, the range of the photocurrent spectrum remained constant for temperatures between $11 \mathrm{~K}$ and $130 \mathrm{~K}$, which means that $E_{L}$ did not vary in this temperature range. Finally, $\mathrm{VBO}=E_{B}-E_{H}-E_{L}<0.18 \mathrm{eV}$.

Note that, in the previous analysis, the InAs wetting layer, which should be present between the $3 \mathrm{~nm} \mathrm{AlGaAs} \mathrm{barrier}$ and the QDs, has been neglected. It is also possible that arsenic atoms have incorporated to the $\mathrm{InGaP}$ spacers during the growth of the AlGaAs barriers [28], forming a quaternary InGaAsP alloy that would also modify the band diagram.

From the previous discussion, the values $E_{G}=1.88$ $\mathrm{eV}, E_{H}=1.26 \mathrm{eV}$ and $E_{L}>0.4 \mathrm{eV}$ are obtained. For comparison, the simplified band diagram of the ideal IBSC is illustrated in Fig. 7 (right). Despite the large bandgap of the host material, the VBO is quite small, as it is found in InAs/AlGaAs-based prototypes [13]. Our device presents a close-to-optimum bandgap distribution from the point of view of theoretical efficiency. However, the measured large value of $E_{L}$ did not come, in our case, with an increase of the activation energy of the carrier thermal escape, which makes this first prototype invalid for proper operation at RT. The break up between $E_{L}$ and $E_{a}$ can be explained by the presence of multiple confined excited levels in the QDs or by the existence of a wetting layer that reduces the effective barrier for carrier escape [29], [30].

From thermodynamical reasons, an electron-hole pair produced by the absorption of two photons, one with energy 


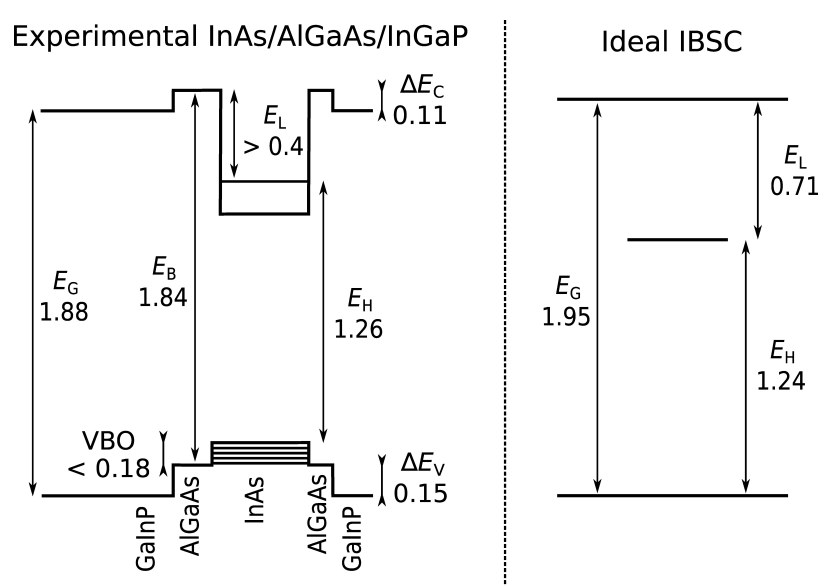

Figure 7: Simplified band diagram at RT of (left) the experimental InAs/AlGaAs/GaInP-QD structure and (right) an ideal IBSC.

$E_{H}$ and another with energy $E_{L}$, cannot be extracted at a voltage higher than $\left(E_{H}+E_{L}\right) / e$, where $e$ is the elementary charge. This sets a fundamental limit to the open-circuit voltage, $V_{O C}$, of the IBSC. In the ideal case - Fig. 7 (right) - $E_{G}=E_{H}+E_{L}$, so the $V_{O C}$ is fundamentally limited by $E_{G}$. As mentioned in the Introduction, GaAs barriers have been previously used to avoid intermixing between InAs QDs and the InGaP spacers and to increase the electron confinement in the QDs. While this has been demonstrated to be effective, the influence of these low bandgap (compared to the spacers) barriers on the $V_{O C}$ has not been discussed. Low bandgap barriers may lead to a fundamental reduction of the limiting $V_{O C}$ (from $E_{G}$ to the bandgap of the barriers, $\left.E_{B}\right)$ [31]. This is why we have decided to use AlGaAs barriers, where $E_{B}(1.84 \mathrm{eV})$ is close to $E_{G}(1.88 \mathrm{eV})$, in order to achieve a good bandgap distribution but avoiding the possibility imposing a lower limit to the $V_{O C}$. Our results confirm that AlGaAs barriers are as effective as GaAs barriers for achieving an adequate bandgap distribution. Further study is needed to evaluate the influence of these barriers on the voltage preservation of the devices.

\section{CONCLUSION}

We have fabricated and characterized wide-bandgap InAs/InGaP QD-IBSCs. Our devices present an improved bandgap distribution compared to other lower-bandgap candidates. The inclusion of thin AlGaAs barriers has served to reduce $E_{H}$ and increase $E_{L}$, both obtained experimentally by photocurrent measurements. The high measured value of $E_{L}>0.4 \mathrm{eV}$ is in contrast with the low measured activation energy of carrier thermal escape, $E_{a}=127 \mathrm{meV}$. This difference indicates the presence of multiple excited states in the CB of the QDs and/or a wetting layer.

\section{ACKNOWLEDGMENT}

The authors would like to thank the Spanish Government for funding through the PROMESA project (grant ENE201237804-C02-01) and UK Engineering and Physics Research Council (EPSRC) through Grant EP/K029118/.

\section{REFERENCES}

[1] A. Luque and A. Martí, "Increasing the efficiency of ideal solar cells by photon induced transitions at intermediate levels," Physical Review Letters, vol. 78, no. 26, pp. 5014-5017, 1997, fisrt proposal of the IBSC.

[2] I. Ramiro, A. Martí, E. Antolín, and A. Luque, "Review of experimental results related to the operation of intermediate band solar cells," Photovoltaics, IEEE Journal of, vol. 4, no. 2, pp. 736-748, 2014.

[3] A. Martí, L. Cuadra, and A. Luque, "Quantum dot intermediate band solar cell," in 28th Photovoltaic Specialists Conference, vol. Conference Record of the Twenty-Eighth IEEE. IEEE, 2000, pp. 940-943.

[4] A. Martí, E. Antolín, C. R. Stanley, C. D. Farmer, N. Lopez, P. Díaz, E. Canovas, P. G. Linares, and A. Luque, "Production of photocurrent due to intermediate-to-conduction-band transitions: a demonstration of a key operating principle of the intermediate-band solar cell," Physical Review Letters, vol. 97, no. 24, pp. 247 701-4, 2006.

[5] P. G. Linares, A. Martí, C. D. Antolín, E.and Farmer, I. Ramiro, C. R. Stanley, and A. Luque, "Voltage recovery in intermediate band solar cells," Solar EnergyMaterials\&SolarCells, vol. 98, pp. 240-244, 2011.

[6] E. Antolín, A. Martí, P. G. Linares, I. Ramiro, E. Hernández, C. D. Farmer, C. R. Stanley, and A. Luque, "Advances in quantum dot intermediate band solar cells," in 35th Photovoltaic Specialists Conference, PVSC 2010. Hawaii, EEUU: IEEE, 20/06/2010 - 25/06/2010 2010, pp. 00065-00070.

[7] E. Antolín, A. Martí, C. D. Farmer, P. G. Linares, E. Hernández, A. M. Sánchez, T. Ben, S. I. Molina, C. R. Stanley, and A. Luque, "Reducing carrier escape in the inas/gaas quantum dot intermediate band solar cell," Journal of Applied Physics, vol. 108, pp. 064513 1-7, 2010.

[8] S. Sauvage, P. Boucaud, F. Julien, J.-M. Gérard, and V. Thierry-Mieg, "Intraband absorption in n-doped inas/gaas quantum dots," Applied Physics Letters, vol. 71, no. 19, pp. 2785-2787, 1997.

[9] S. Sauvage, P. Boucaud, F. Glotin, R. Prazeres, J.-M. Ortega, A. Lematre, J.-M. Gérard, and V. Thierry-Flieg, "Saturation of intraband absorption and electron relaxation time in n-doped inas/gaas self-assembled quantum dots," Applied Physics Letters, vol. 73, no. 26, pp. 3818-3821, 1998.

[10] W. Ng, E. Zibik, L. Wilson, M. Skolnick, J. Cockburn, and M. Steer, "Tuning of intraband absorption and photoresponse in self-assembled inas/ gaas quantum dots by thermal annealing," Journal of Applied Physics, vol. 103, no. 6, p. 066101, 2008.

[11] P. G. Linares, A. Martí, E. Antolín, and A. Luque, "Iii-v compound semiconductor screening for implementing quantum dot intermediate band solar cells," Journal of Applied Physics, vol. 109, p. 014313, 2011.

[12] I. Ramiro, E. Antolín, M. Steer, P. Linares, E. Hernandez, I. Artacho, E. Lopez, T. Ben, J. Ripalda, and S. Molina, "Inas/algaas quantum dot intermediate band solar cells with enlarged sub-bandgaps," in Photovoltaic Specialists Conference (PVSC), 2012 38th IEEE. IEEE, 2012, pp. 000652-000656.

[13] E. Antolín, I. Ramiro, P. G. Linares, E. López, I. Artacho, A. Datas, A. Martí, A. Luque, M. J. Steer, and C. R. Stanley, (To be published).

[14] V. Popescu and A. Zunger, "Three-dimensional assemblies of semiconductor quantum dots in a wide-gap matrix providing an intermediate band for absorption," Journal of Applied Physics, vol. 112, no. 11, p. 114320, 2012.

[15] H. Amanai, S. Nagao, and H. Sakaki, "Inas/gainp self-assembled quantum dots: molecular beam epitaxial growth and optical properties," Journal of Crystal Growth, vol. 227, pp. 1089-1094, 2001.

[16] T. Sugaya, R. Oshima, K. Matsubara, and S. Niki, "In (ga) as quantum dots on ingap layers grown by solid-source molecular beam epitaxy," Journal of Crystal Growth, vol. 378, pp. 430-434, 2013.

[17] D. V. Forbes, A. M. Podell, M. A. Slocum, S. J. Polly, and S. M. Hubbard, "Omvpe of inas quantum dots on an ingap surface," Materials Science in Semiconductor Processing, vol. 16, no. 4, pp. 1148-1153, 2013.

[18] A. Mellor, A. Luque, I. Tobías, and A. Martí, "The influence of quantum dot size on the sub-bandgap intraband photocurrent in intermediate band solar cells," Applied Physics Letters, vol. 101, no. 13, p. 133909, 2012.

[19] T. Sugaya, A. Takeda, R. Oshima, K. Matsubara, S. Niki, and Y. Okano, "Ingap-based ingaas quantum dot solar cells with gaas spacer layer fabricated using solid-source molecular beam epitaxy," Applied Physics Letters, vol. 101, no. 13, pp. 133110-133110-4, 2012.

[20] H. Liu, I. Sellers, T. Badcock, D. Mowbray, M. Skolnick, K. Groom, M. Gutierrez, M. Hopkinson, J. Ng, J. David et al., "Improved performance of $1.3 \mu \mathrm{m}$ multilayer inas quantum-dot lasers using a highgrowth-temperature gaas spacer layer," Applied Physics Letters, vol. 85, no. 5 , pp. 704-706, 2004 
[21] A. Martí, E. Antolín, E. Cánovas, N. López, P. G. Linares, A. Luque, C. R. Stanley, and C. D. Farmer, "Elements of the design and analysis of quantum-dot intermediate band solar cells," Thin solid films, vol. 516, no. 20, pp. 6716-6722, 2008.

[22] A. Martí Vega, E. Antolín Fernández, P. García-Linares Fontes, I. Ramiro González, E. López Estrada, I. Tobías Galicia, and A. Luque López, "A puzzling solar cell structure: an exercise to get insight on intermediate band solar cells," in Photovoltaic Specialists Conference (PVSC), 2013 39th IEEE. IEEE, 2013.

[23] A. Martí, N. Lopez, E. Antolín, E. Canovas, A. Luque, C. R. Stanley, C. D. Farmer, and P. Díaz, "Emitter degradation in quantum dot intermediate band solar cells," Applied Physics Letters, vol. 90, no. 23, pp. 233510-3, 2007.

[24] P. W. Fry, I. E. Itskevich, S. R. Parnell, J. J. Finley, L. R. Wilson, K. L. Schumacher, D. J. Mowbray, M. S. Skolnick, M. Al-Khafaji, and A. G. Cullis, "Photocurrent spectroscopy of inas/gaas self-assembled quantum dots," Physical Review B, vol. 62, no. 24, pp. 16784-16791, 2000, thermal activation energy InAs/GaAs measured by photocurrent.

[25] R. Tamaki, Y. Shoji, Y. Okada, and K. Miyano, "Spectrally resolved interband and intraband transitions by two-step photon absorption in ingaas/gaas quantum dot solar cells," in Photovoltaic Specialists Conference (PVSC), 2014 40th IEEE. IEEE, 2014.

[26] R. L. Anderson, "Germanium-gallium arsenide heterojunctions," IBM J. Res. Dev., vol. 4, no. 3, pp. 283-287, 1960.

[27] Y.-H. Cho, B.-D. Choe, and H. Lim, "Band offset transitivity in algaas/ingap/ingaasp heterostructures on a gaas substrate," Applied Physics Letters, vol. 69, no. 24, pp. 3740-3742, 1996.

[28] O. Schuler, O. Dehaese, X. Wallart, and F. Mollot, "Interface quality and electron transfer at the gainp on gaas heterojunction," Journal of applied physics, vol. 84, no. 2, pp. 765-769, 1998.

[29] S. Sanguinetti, M. Henini, M. G. Alessi, M. Capizzi, P. Frigeri, and S. Franchi, "Carrier thermal escape and retrapping in self-assembled quantum dots," Physical Review B, vol. 60, no. 11, p. 8276, 1999, experimental and model of carrier dynamics in QDs.

[30] E. Le Ru, J. Fack, and R. Murray, "Temperature and excitation density dependence of the photoluminescence from annealed inas/gaas quantum dots," Physical Review B, vol. 67, no. 24, p. 245318, 2003, fantastic quantum dot carrier dynamics study.

[31] P. Linares, E. López, I. Ramiro, A. Datas, E. Antolín, Y. Shoji, T. Sogabe, Y. Okada, A. Martí, and A. Luque, "Voltage limitation analysis in strain-balanced inas/gaasn quantum dot solar cells applied to the intermediate band concept," Solar Energy Materials and Solar Cells, vol. 132, pp. 178-182, 2015. 